\section{Changing landscape of immunosuppression in ANCA-associated vasculitis}

Over last decades, there have been substantial advances in the immunosuppressive treatment for antineutrophil cytoplasmic antibody (ANCA)-associated vasculitides (AAV), that is, granulomatosis with polyangiitis (GPA) and microscopic polyangiitis (MPA). The recently published MYCYC study added another piece of evidence to our knowledge of treatment options in patients with AAV. ${ }^{1}$ In an open-label, two-group, paralleldesign, randomised trial, 140 newly diagnosed patients with active GPA or MPA were allocated to remission induction with mycophenolate mofetil (MMF) or pulsed cyclophosphamide in combination with the same oral glucocorticoid regimen. Non-inferiority was demonstrated for the primary remission endpoint, which occurred by 6 months in $67 \%$ of patients in the MMF group and $61 \%$ of patients in the cyclophosphamide group (risk difference $5.7 \%, 90 \%$ CI $-7.5 \%$ to $19 \%$ ). The median time to remission was also similar in the MMF and cyclophosphamide groups. However, treatment with MMF was associated with a higher occurrence of relapses compared with cyclophosphamide ( $33 \%$ vs $19 \%, \mathrm{p}=0.049$ ). As expected, the higher relapse rate following remission induction with MMF was accounted for by more frequent relapses in patients with proteinase-3 ANCA, but not patients with myeloperoxidase (MPO) ANCA. The authors suggested that MMF induction therapy might be a suitable alternative to cyclophosphamide in patients at low risk of relapse, such as those with MPO-ANCA.

Unlike methotrexate, MMF can be used in patients with moderate or severe renal disease. Moreover, remission induction treatment with MMF was similar in efficacy to pulsed cyclophosphamide in patients with lupus nephritis. ${ }^{2}$ It seems that MPO-ANCA associated renal vasculitis may be a primary indication for administration of MMF. At entry into MYCYC study, $81 \%$ of patients in the MMF and cyclophosphamide groups presented with proteinuria, haematuria and/or impaired kidney function. Only two patients in both groups progressed to end stage renal disease, while average estimated glomerular filtration rate at 18 months did not differ between groups.

Unlike cyclophosphamide, MMF does not induce infertility that causes particular concerns in younger patients with AAV. Therefore, adolescents and young adults should also be considered as candidates for MMF induction regimen. MYCYC study was the first randomised trial in AAV to include paediatric patients $(n=8)$. The response rates were similar in the MMF and cyclophosphamide groups in children. However, the small number of paediatric participants precludes any definite conclusions regarding the efficacy or safety of MMF in this population. Of note, an advantage of MMF in safety over cyclophosphamide should not be overestimated given the similar rates of adverse events, including serious infections, in the two groups. Over 18 months of follow-up, malignancies were reported in one patient from MMF group and one patient from cyclophosphamide group.

Only patients with newly diagnosed AAV participated in the MYCYC study. Therefore, the efficacy of MMF as compared with cyclophosphamide in patients with relapsing AAV is not established. In RAVE trial, conventional immunosuppression with cyclophosphamide followed by azathioprine was inferior to rituximab at 6 and 12 months in 101 patients who had relapsing disease at baseline. ${ }^{3}$ We can speculate that in patients with relapsing $\mathrm{AAV}$ remission induction treatment with MMF
Table 1 Possible tailored regimens of remission induction treatment in patients with AAV

\begin{tabular}{lll}
\hline & Non-severe AAV & Severe AAV \\
\hline Proteinase-3 ANCA & Methotrexate* or rituximab & $\begin{array}{l}\text { Rituximabt or } \\
\text { cyclophosphamide }\end{array}$ \\
MPO-ANCA & $\begin{array}{l}\text { Methotrexate* or MMF or } \\
\text { rituximab }\end{array}$ & $\begin{array}{l}\text { Cyclophosphamide or } \\
\text { rituximabt or MMF† }\end{array}$ \\
ANCA-negative & $\begin{array}{l}\text { Methotrexate* or } \\
\text { cyclophosphamide }\end{array}$ & Cyclophosphamide \\
\hline
\end{tabular}

Immunosuppressive medications should be used in combination with glucocorticoids (at least $40 \mathrm{mg}$ daily of prednisone in non-severe AAV and at least $60 \mathrm{mg}$ daily of prednisone in severe AAV).

*Only in patients with estimated glomerular filtration rate $>30 \mathrm{~mL} / \mathrm{min} / 1.73 \mathrm{~m}^{2}$. †Preferably in younger patients as well as in patients with relapsing disease. AAV, ANCA-associated vasculitides; ANCA, antineutrophil cytoplasmic antibody; MMF, mycophenolate mofetil; MPO, myeloperoxidase.

would be even less effective and should be avoided. In the IMPROVE trial, MMF was also inferior to azathioprine for maintaining disease remission in 156 patients with $\mathrm{AAV}^{4}$

Currently, rituximab is increasingly used for initial induction remission treatment in patients with both de novo and relapsing AAV. Moreover, in the USA, rituximab label has been recently updated to include information for maintenance therapy in adult patients with GPA and MPA who have achieved remission with induction treatment. The apparent benefits of rituximab question the future of MMF in patients with AAV. High cost of rituximab is one of the main factors limiting its use in AAV in many countries. The independent Evidence Review Group commissioned by the National Institute for Health and Care Excellence (NICE) has recently concluded that rituximab was likely to represent a costeffective addition to the treatment sequence if given after cyclophosphamide treatment. ${ }^{5}$ In Russia, there is almost a 7 -fold difference between the acquisition costs for rituximab and MMF. However, the emergence of less expensive (by up to $30 \%$ ) biosimilars of rituximab improved patients' access to modern care. Accumulating evidence suggest that a proportion of patients with AAV are overtreated with rituximab and may benefit from safer and more cost-effective low-dose regimens of rituximab administration. ${ }^{6}$

In summary, the results of MYCYC study and other randomised controlled trials pave the way for precision medicine in AAV. We agree with the authors that stratified treatment approaches are indicated in order to optimise long-term outcomes. The choice of treatment depends on many factors, including age of patient, severity of AAV, ANCA specificity, renal function, the presence of granulomatous lesions and so on (table 1). Emerging treatment options, that is, complement inhibitors (avacopan, IFX-1) may result in implementation of safer, steroid-free remission induction and maintenance regimens.

\section{Sergey V Moiseev $\odot,{ }^{1}$ Ilya Smitienko, ${ }^{2}$ Nikolai Bulanov, ${ }^{1}$ Ekaterina Karovaikina, ${ }^{1}$ Pavel I Novikov ${ }^{1}$}

${ }^{1}$ Department of Rheumatology, Tareev Clinic of Internal Diseases, Sechenov First Moscow State Medical University, Moscow, Russian Federation

${ }^{2}$ Medical Center K+31, Moscow, Russian Federation

Correspondence to Professor Sergey V Moiseev, Tareev Clinic of Internal Diseases, Sechenov First Moscow State Medical University, Moscow 119435, Russian Federation; clinpharm@mtu-net.ru

Correction notice This article has been corrected since it published Online First. Table 1 has been corrected.

Handling editor Josef S Smolen 
Contributors All authors participated in the preparation of the manuscript.

Funding Russian Academic Excellence Project 5-100.

Competing interests None declared.

Patient consent for publication Not required.

Provenance and peer review Not commissioned; internally peer reviewed.

(c) Author(s) (or their employer(s)) 2020. No commercial re-use. See rights and permissions. Published by BMJ.

\section{(D) Check for updates}

To cite Moiseev SV, Smitienko I, Bulanov N, et al. Ann Rheum Dis 2020;79:e59.

Received 24 January 2019

Accepted 26 January 2019

Published Online First 12 February 2019

Ann Rheum Dis 2020;79:e59. doi:10.1136/annrheumdis-2019-215123
ORCID iD

Sergey V Moiseev http://orcid.org/0000-0002-7232-4640

\section{REFERENCES}

1 Jones RB, Hiemstra TF, Ballarin J, et al. Mycophenolate mofetil versus cyclophosphamide for remission induction in ANCA-associated vasculitis: a randomised, non-inferiority trial. Ann Rheum Dis 2019:78:399-405.

2 Ginzler EM, Dooley MA, Aranow C, et al. Mycophenolate mofetil or intravenous cyclophosphamide for lupus nephritis. N Engl J Med 2005;353:2219-28.

3 Specks U, Merkel PA, Seo P, et al. Efficacy of remission-induction regimens for ANCAassociated vasculitis. N Engl J Med 2013;369:417-27.

4 Hiemstra TF, Walsh M, Mahr A, et al. Mycophenolate mofetil vs azathioprine for remission maintenance in antineutrophil cytoplasmic antibody-associated vasculitis: a randomized controlled trial. JAMA 2010;304:2381-8.

5 Latimer NR, Carroll C, Wong R, et al. Rituximab in combination with corticosteroids for the treatment of anti-neutrophil cytoplasmic antibody-associated vasculitis: a NICE single technology appraisal. Pharmacoeconomics 2014;32:1171-83.

6 Moiseev SV, Bulanov NM, Zykova AS, et al. Rituximab in ANCA-associated vasculitis: Fewer infusions or ultra low-dose maintenance therapy? Ann Rheum Dis 2019;78:e99. 